Supporting information

\title{
Selenate and Nitrate Bio-reductions Using Methane as the Electron Donor in a Membrane Biofilm Reactor
}

Chun-Yu Lai ${ }^{1,2,3}$, Li-Lian Wen ${ }^{1}$, Ling-Dong Shi $^{1}$, Kan-Kan Zhao ${ }^{1}$, Yi-Qi Wang ${ }^{1}$, Xiaoe Yang ${ }^{2}$, Bruce E. Rittmann ${ }^{4}$, Chen Zhou $^{4}$, Youneng Tang ${ }^{5}$, Ping Zheng ${ }^{1,3}$, He-Ping Zhao ${ }^{1,3, *}$

1. Department of Environmental Engineering, College of Environmental and Resource Science, Zhejiang University, Hangzhou, China.

2. Ministry of Education Key Laboratory of Environmental Remediation and Ecological Health, College of Environmental and Resource Sciences, Zhejiang University, Hangzhou 310058, China

3. Zhejiang Province Key Lab Water Pollut Control \& Envi, Zhejiang University, Hangzhou, Zhejiang, China.

4. Swette Center for Environmental Biotechnology, Biodesign Institute at Arizona State University, P.O. Box 875701, Tempe, Arizona 85287-5701, U.S.A.

5. Department of Civil and Environmental Engineering, FAMU-FSU College of Engineering, Florida State University, Tallahassee, Florida, 32310-6046, U.S.A.

* Correspondence to Dr. He-Ping Zhao. Tel: 0086-571-88982739, Fax: 0086-571-88982907, E-mail: zhaohp@,zju.edu.cn 


\section{The calculations for overall-reaction stoichiometry}

The following development of overall-reaction stoichiometry follows the procedures of Rittmann and McCarty (2001). The energy reaction involves half reactions of the electron acceptor $\left(\mathrm{SeO}_{4}{ }^{2-}\right)$ and electron donor $\left(\mathrm{CH}_{4}\right)$ :

$\mathrm{R}_{\mathrm{a}}: \quad 1 / 6 \mathrm{SeO}_{4}{ }^{2-}+\mathrm{e}^{-}+4 / 3 \mathrm{H}^{+} \rightarrow 1 / 6 \mathrm{Se} 0+2 / 3 \mathrm{H}_{2} \mathrm{O}$

$\mathrm{R}_{\mathrm{d}}: \quad 1 / 8 \mathrm{CH}_{4}+1 / 4 \mathrm{H}_{2} \mathrm{O} \rightarrow 1 / 8 \mathrm{CO}_{2}+\mathrm{H}^{+}+\mathrm{e}^{-}$

Thus, the overall energy reaction is:

$\mathrm{R}_{\mathrm{e}}: \quad 1 / 6 \mathrm{SeO}_{4}{ }^{2-}+1 / 3 \mathrm{H}^{+}+1 / 8 \mathrm{CH}_{4} \rightarrow 1 / 8 \mathrm{CO}_{2}+1 / 6 \mathrm{Se}^{0}+5 / 12 \mathrm{H}_{2} \mathrm{O}$

The synthesis reaction with $\mathrm{NO}_{3}{ }^{-}$as the $\mathrm{N}$ sources is:

$\mathrm{R}_{\mathrm{s}}: 1 / 28 \mathrm{NO}_{3}{ }^{-}+1 / 8 \mathrm{CH}_{4}+3 / 56 \mathrm{CO}_{2}+1 / 28 \mathrm{H}^{+} \rightarrow 1 / 28 \mathrm{C}_{5} \mathrm{H}_{7} \mathrm{O}_{2} \mathrm{~N}+1 / 7 \mathrm{H}_{2} \mathrm{O}$

The partitioning of electrons from the donor to the synthesis reaction is with fraction $\mathrm{f}_{\mathrm{s}}$, and the fraction to the acceptor being $\mathrm{f}_{\mathrm{e}}=1-\mathrm{f}_{\mathrm{s}}$. Relevant values are:

$\mathrm{f}_{\mathrm{s}}=0.333, \mathrm{f}_{\mathrm{e}}=0.667$, which makes the net overall reaction:

$\mathrm{SeO}_{4}{ }^{2-}+1.124 \mathrm{CH}_{4}+0.107 \mathrm{NO}_{3}{ }^{-}+2.104 \mathrm{H}^{+} \rightarrow \mathrm{Se}^{0}+0.59 \mathrm{CO}_{2}+0.107 \mathrm{C}_{5} \mathrm{H}_{7} \mathrm{O}_{2} \mathrm{~N}+$ $2.929 \mathrm{H}_{2} \mathrm{O}$

Following the same procedure give the net overall reactions for methane oxidation with respiration of nitrate $\left(f_{s}=333\right)$ and oxygen $\left(f_{s}=0.667\right)$ :

$\mathrm{NO}_{3}{ }^{-}+1.2 \mathrm{CH}_{4}+\mathrm{H}^{+} \rightarrow 0.2 \mathrm{CO}_{2}+0.4 \mathrm{~N}_{2}+0.2 \mathrm{C}_{5} \mathrm{H}_{7} \mathrm{O}_{2} \mathrm{~N}+2.2 \mathrm{H}_{2} \mathrm{O}$

$\mathrm{O}_{2}+1.25 \mathrm{CH}_{4}+0.214 \mathrm{NO}_{3}{ }^{-}+0.214 \mathrm{H}^{+} \rightarrow 0.179 \mathrm{CO}_{2}+1.86 \mathrm{H}_{2} \mathrm{O}+0.214 \mathrm{C}_{5} \mathrm{H}_{7} \mathrm{O}_{2} \mathrm{~N}(7)$

For comparison, the reaction stoichiometry neglecting electron-donor consumption for biomass synthesis.

$$
\begin{aligned}
& 1 / 6 \mathrm{SeO}_{4}{ }^{2-}+1 / 8 \mathrm{CH}_{4}+1 / 3 \mathrm{H}^{+} \rightarrow 1 / 8 \mathrm{CO}_{2}+1 / 6 \mathrm{Se}+5 / 12 \mathrm{H}_{2} \mathrm{O} \\
& 1 / 5 \mathrm{NO}_{3}{ }^{-}+1 / 8 \mathrm{CH}_{4}+1 / 5 \mathrm{H}^{+} \rightarrow 1 / 8 \mathrm{CO}_{2}+1 / 10 \mathrm{~N}_{2}+7 / 20 \mathrm{H}_{2} \mathrm{O} \\
& 1 / 4 \mathrm{O}_{2}+1 / 8 \mathrm{CH}_{4} \rightarrow 1 / 8 \mathrm{CO}_{2}+1 / 4 \mathrm{H}_{2} \mathrm{O}
\end{aligned}
$$


Table S1. Ln(OR) value of predictive specific genes in MBfR biofilms compared to those in the inoculum. Positive values indicate over-representation, and negative values indicate under-representation.

\begin{tabular}{|c|c|c|c|c|c|}
\hline & $\begin{array}{c}\text { \#OTU } \\
\text { ID }\end{array}$ & KEGG_Description & Stage 1 & Stage 2 & Stage 3 \\
\hline \multirow{8}{*}{ 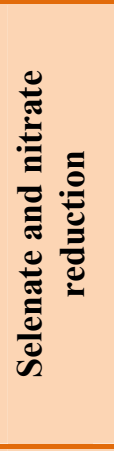 } & K12529 & putative selenate reductase FAD-binding subunit & -0.40 & 0.19 & 0.99 \\
\hline & K00370 & nitrate reductase 1 , alpha subunit & 0.22 & 0.68 & 0.56 \\
\hline & K00371 & nitrate reductase 1 , beta subunit & 0.17 & 0.54 & 0.00 \\
\hline & K00373 & nitrate reductase 1 , delta subunit & 0.67 & 1.02 & 1.01 \\
\hline & K00374 & nitrate reductase 1 , gamma subunit & 0.40 & 0.45 & 0.52 \\
\hline & K00363 & nitrite reductase $(\mathrm{NAD}(\mathrm{P}) \mathrm{H})$ small subunit & 0.47 & 0.29 & 0.01 \\
\hline & K00366 & ferredoxin-nitrite reductase & -0.34 & -0.80 & 0.29 \\
\hline & K00363 & nitrite reductase $(\mathrm{NAD}(\mathrm{P}) \mathrm{H})$ small subunit & 0.47 & 0.29 & 0.01 \\
\hline \multirow{5}{*}{ 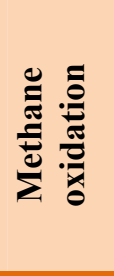 } & K08684 & methane monooxygenase & 0.92 & 1.05 & 0.51 \\
\hline & K14028 & methanol dehydrogenase (cytochrome c) subunit 1 & 0.91 & 0.56 & 0.15 \\
\hline & K14029 & methanol dehydrogenase (cytochrome c) subunit 2 & 0.91 & 0.56 & 0.15 \\
\hline & K10713 & formaldehyde-activating enzyme & 0.91 & 0.62 & 0.20 \\
\hline & K00124 & formate dehydrogenase, beta subunit & 0.08 & 0.10 & -0.07 \\
\hline \multirow{5}{*}{ 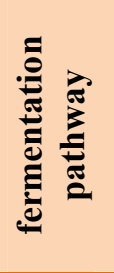 } & K00925 & acetate kinase & 0.10 & 0.18 & 0.06 \\
\hline & K01782 & 3-hydroxyacyl-CoA dehydrogenase / enoyl-CoA hydratase / & 0.38 & 0.22 & 0.65 \\
\hline & K00024 & malate dehydrogenase & 0.17 & 0.22 & 0.33 \\
\hline & K00239 & succinate dehydrogenase flavoprotein subunit & 0.00 & 0.10 & 0.19 \\
\hline & K00016 & L-lactate dehydrogenase & 1.32 & 1.50 & 1.51 \\
\hline
\end{tabular}




\begin{tabular}{|c|c|c|c|c|c|}
\hline & K01632 & fructose-6-phosphate phosphoketolase & 1.33 & 0.98 & -0.29 \\
\hline & K01662 & 1-deoxy-D-xylulose-5-phosphate synthase & 0.18 & 0.15 & 0.05 \\
\hline & K13788 & phosphate acetyltransferase & 1.49 & 1.11 & 0.40 \\
\hline & K01676 & fumarate hydratase, class I & 0.43 & 0.33 & 0.03 \\
\hline & K00031 & isocitrate dehydrogenase & -0.01 & 0.21 & 0.28 \\
\hline & K14127 & F420-non-reducing hydrogenase iron-sulfur subunit D & 1.88 & -1.19 & -0.71 \\
\hline \multirow{5}{*}{ 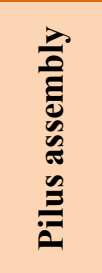 } & K02279 & pilus assembly protein $\mathrm{CpaB}$ & 0.26 & 0.39 & 0.47 \\
\hline & K02280 & pilus assembly protein $\mathrm{CpaC}$ & 0.23 & 0.33 & 0.32 \\
\hline & K02281 & pilus assembly protein $\mathrm{CpaD}$ & -1.90 & -1.39 & -0.39 \\
\hline & K02282 & pilus assembly protein $\mathrm{CpaE}$ & 0.25 & 0.02 & 0.15 \\
\hline & K02283 & pilus assembly protein $\mathrm{CpaF}$ & 0.39 & 0.15 & 0.33 \\
\hline \multirow{6}{*}{ 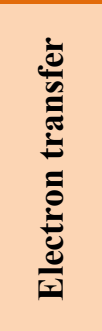 } & K00330 & NADH-quinone oxidoreductase subunit A ;NADH dehydrogenase I subunit A & -0.02 & 0.38 & 0.73 \\
\hline & K00331 & NADH dehydrogenase I subunit B];NADH-quinone oxidoreductase subunit B & -0.08 & 0.32 & 0.66 \\
\hline & K00332 & NADH-quinone oxidoreductase subunit $\mathrm{C}$;NADH dehydrogenase I subunit $\mathrm{C}$ & -0.43 & -0.02 & 0.29 \\
\hline & K03612 & electron transport complex protein $\mathrm{RnfG}$ & 0.74 & 0.62 & 0.15 \\
\hline & K03613 & electron transport complex protein $\mathrm{RnfE}$ & 0.75 & 0.62 & 0.16 \\
\hline & K03614 & electron transport complex protein $\mathrm{RnfD}$ & 0.75 & 0.62 & 0.16 \\
\hline \multirow{3}{*}{ ن } & K03586 & cell division protein FtsL & 0.23 & 0.02 & 0.04 \\
\hline & K03587 & cell division protein FtsI (penicillin-binding protein 3) & 0.11 & 0.20 & 0.29 \\
\hline & K09888 & cell division protein ZapA & 0.98 & 0.80 & 0.56 \\
\hline
\end{tabular}




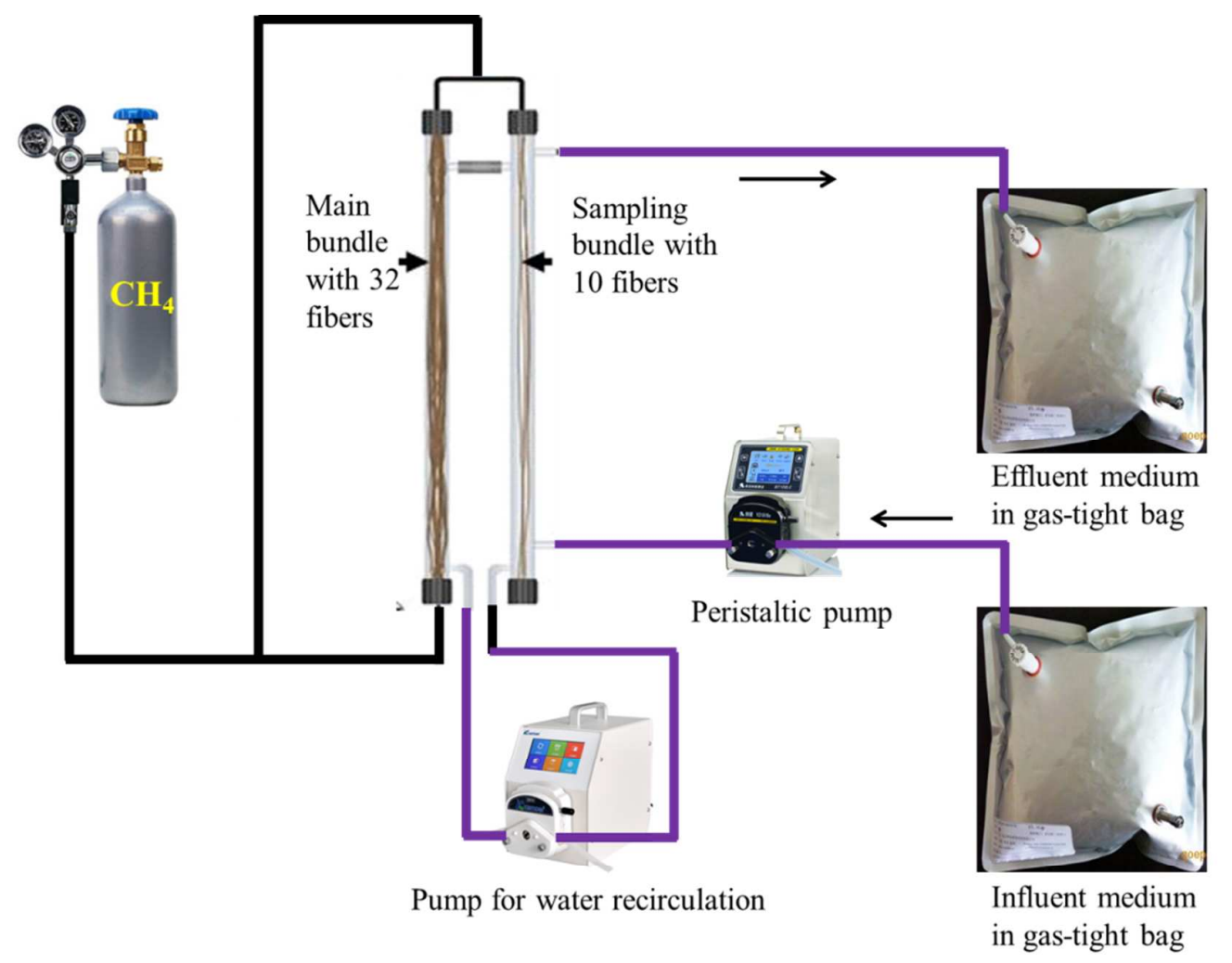

Figure S1. Schematic of the $\mathrm{CH}_{4}$-based MBfR experimental system. 


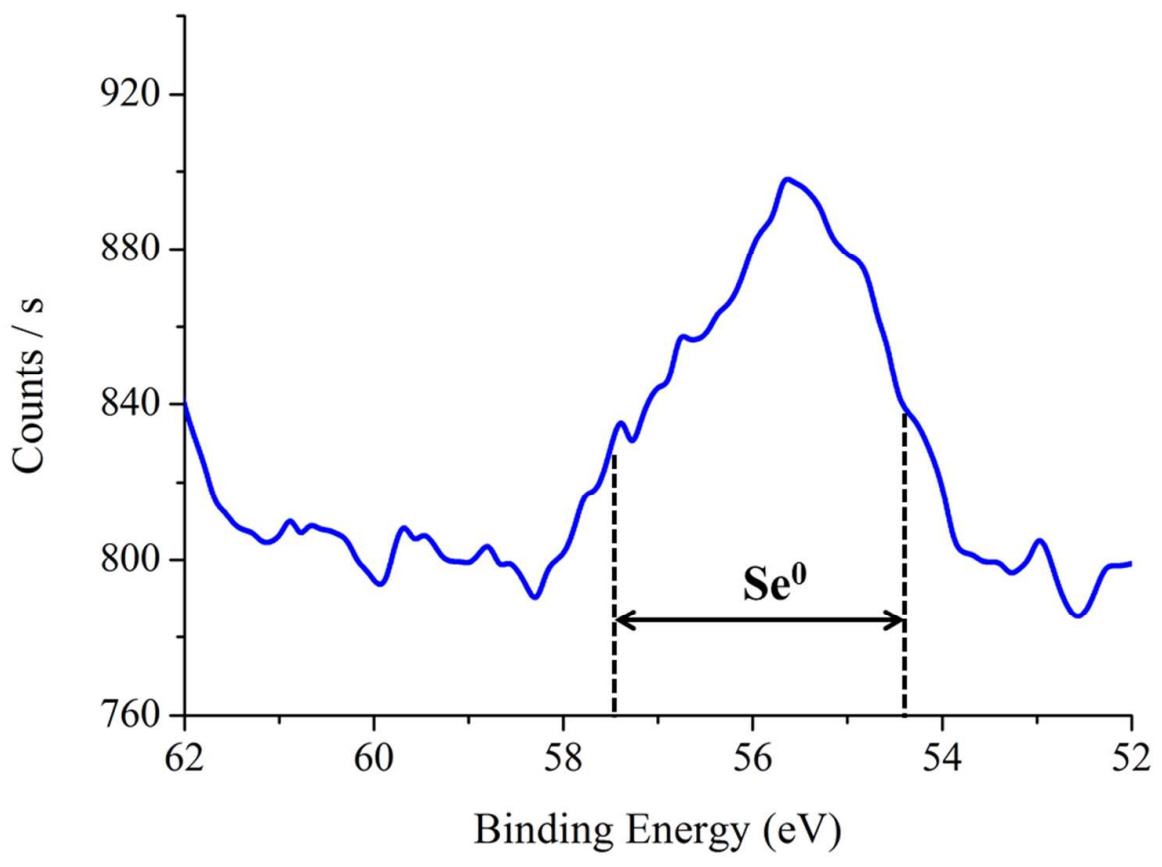

Figure S2. Se 3D XPS of MBfR biofilms (smoothed by XPS software origin 7.5).

The spectrum profiles indicate that $\mathrm{Se}^{0}$ was the main Selenium constituent in the precipitates in the $\mathrm{CH}_{4}$-based MBfR. The energy range for $\mathrm{Se}^{0}$ is marked according to Li et al (2014). 


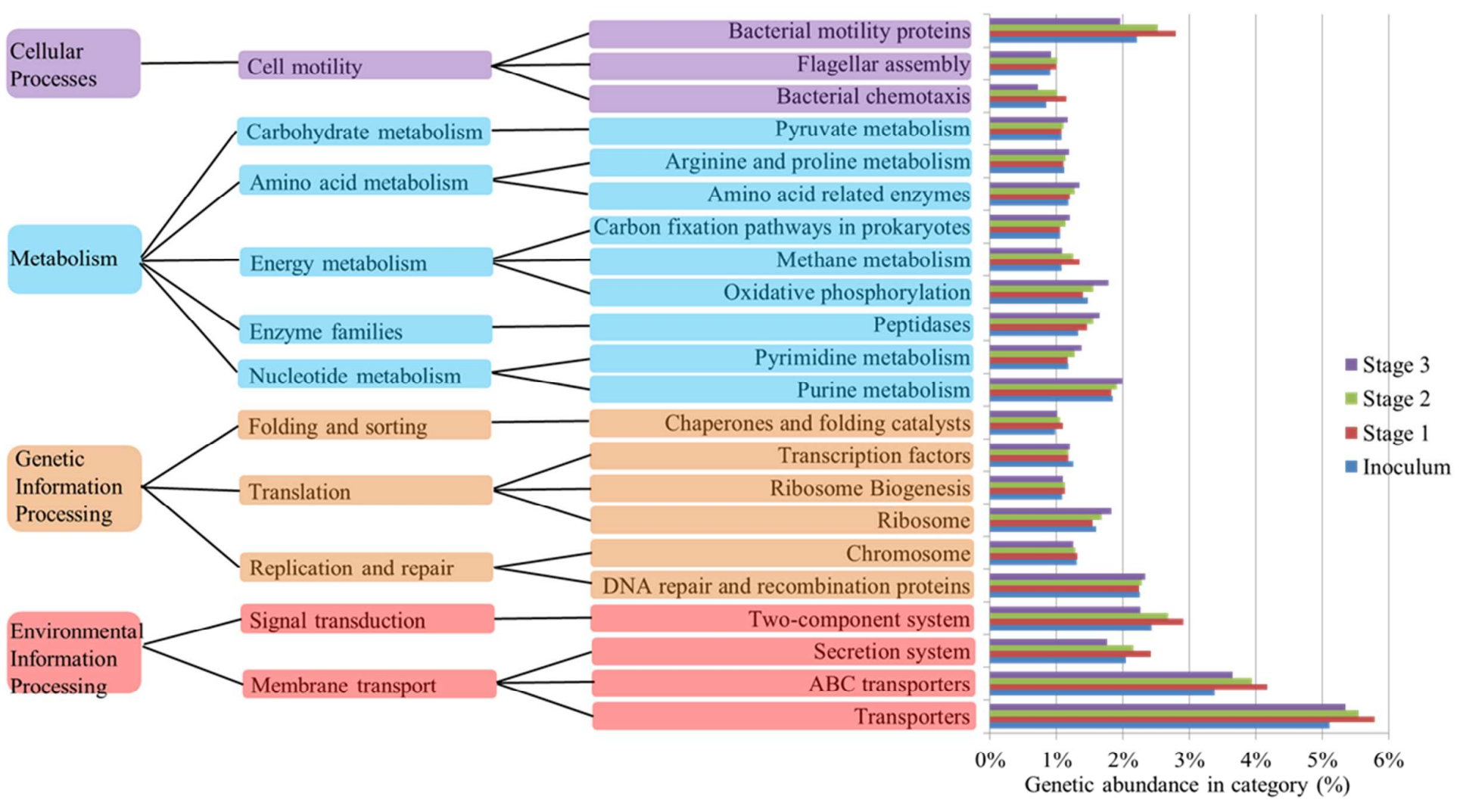

Figure S3. Predicted functional-gene composition of the metagenome, based on PICRUSt analysis, for the inoculum and the biofilms of all stages. Genes $<1 \%$ and with unknown function are not included. The genes in the inoculum were reported previously by Lai et al (2016). ${ }^{22}$ 


\section{References:}

Li, B. B.; Cheng, Y. Y.; Wu, C.; Li, W. W.; Li, N.; Yang, Z. C.; Tong, Z. H.; Yu, H. Q. Selenite reduction by Shewanella oneidensis MR-1 is mediated by fumarate reductase in periplasm. Sci. Rep. 2014, 3735, 1-6.

Rittmann, B. E.; McCarty, P. L. Environmental Biotechnology: Principles and Applications; McGraw-Hill Book Co.: New York, 2001. 